\title{
Changes in variance of neuronal signals may be perceptually relevant for stereo vision
}

\author{
Nela Cicmil \\ Department of Physiology, \\ Anatomy \& Genetics \\ University of Oxford \\ Oxford, OX1 3PT, U.K. \\ nela.cicmil@dpag.ox.ac.uk
}

\author{
Andrew J. Parker \\ Department of Physiology, \\ Anatomy \& Genetics \\ University of Oxford \\ Oxford, OX1 3PT, U.K. \\ andrew.parker@dpag.ox.ac.uk
}

\author{
Kristine Krug \\ Department of Physiology, \\ Anatomy \& Genetics \\ University of Oxford \\ Oxford, OX1 3PT, U.K. \\ kristine.krug@dpag.ox.ac.uk
}

\begin{abstract}
We measured the variance/mean $(\mathrm{v} / \mathrm{m})$ ratio of neuronal firing rates in visual areas $\mathrm{V} 1, \mathrm{~V} 2$ and $\mathrm{V} 5 / \mathrm{MT}$ in response to correlated and anti-correlated random dot stereograms. Disparity-selective neurons in early visual areas V1 and V2 showed no significant difference in $\mathrm{v} / \mathrm{m}$ ratios to the two types of stereo-stimuli, but neurons in area V5/MT had a significantly greater $\mathrm{v} / \mathrm{m}$ ratio for anti-correlated compared to correlated stimuli. These results demonstrate that neurons in a visual area higher in the cortical hierarchy have a greater response variability to anti-correlated stimuli, which do not give rise to a coherent stereo percept. A recurrent cortical network including V5/MT that quenches neural variability may contribute to solving the stereo correspondence problem.
\end{abstract}

\section{Categories and Subject Descriptors}

I.4.8 [Image Processing and Computer Vision]: Scene Analysis-Stereo; J.3.8 [Life and Medical Sciences]: Biology and Genetics

\section{General Terms}

Measurement, Performance, Experimentation

\section{Keywords}

Visual Cortex, Neural Variability, Stereo Correspondence, Depth Perception

\section{INTRODUCTION}

Comparing the images on the two retinas is an important stage of neural processing that enables the visual system to infer three-dimensional information about the environment. Since the eyes view the world from slightly different locations, these retinal images are not identical. Stereopsis is the ability to judge depth based on binocular disparities, the differences in locations of corresponding features on the retinas. Neurons selective for specific binocular disparities can be found throughout the visual cortex [10, 4, 9]. Matching corresponding features in the two retinal images correctly is known as the correspondence problem, which neurophysiological evidence suggests is progressively solved as one moves from primary visual cortex along the visual cortical hierarchy [9]. However, the mechanisms by which networks of neurons in visual cortex reject false matches and solve the correspondence problem have not been fully characterised.

The underlying mechanisms of stereopsis can be probed by challenging disparity-selective neurons with stimuli that contain binocular disparities but do not yield a consistent depth percept. One such stimulus is the anti-correlated randomdot stereogram (AC-RDS) [2]. In AC-RDS, dots in the right eye's image correspond to dots with the opposite contrast in the left eye's image, so the AC-RDS does not allow a global solution to the correspondence problem and does not give rise to a perception of stereoscopic depth (Figure 1A). Local filtering models of disparity detection [8] predict that mean firing rates of neurons in primary visual cortex (area V1) signal the disparity of AC-RDS with a phase shift compared to the response to correlated stereograms (C-RDS) [2]. V1 neurons can therefore be described by a local filtering model, which is consistent with their responses to false matches.

Higher in the cortical visual hierarchy, in area V5/MT, it has been proposed that neurons contribute directly to binocular depth judgments since their firing correlates with animals' subjective perception of depth in ambiguous stimuli [3]. Furthermore, electrical microstimulation of area V5/MT biases depth judgments towards the disparity selectivity of the stimulated neurons [5]. However, like in V1, neural responses of $\mathrm{V} 5 / \mathrm{MT}$ cells are also systematically modulated by AC-RDS disparities, suggesting that if these neurons contribute to depth judgments, they do so in a task-specific way, perhaps involving a top-down perceptual signal [6].

Although responses of sensory neurons are typically characterized by their mean firing rate, it is increasingly recognized that the effect of the stimulus on firing response variability can reveal additional information about the role of a neuron in sensory processing, including the cortical network in which the neuron is located [1]. Across cortical areas and species, the onset of a stimulus causes a decline in the variance/mean ratio of neuronal firing (also known as the Fano factor), suggesting that stabilization by sensory input might be a general property of cortex [1]. A decrease in variability 

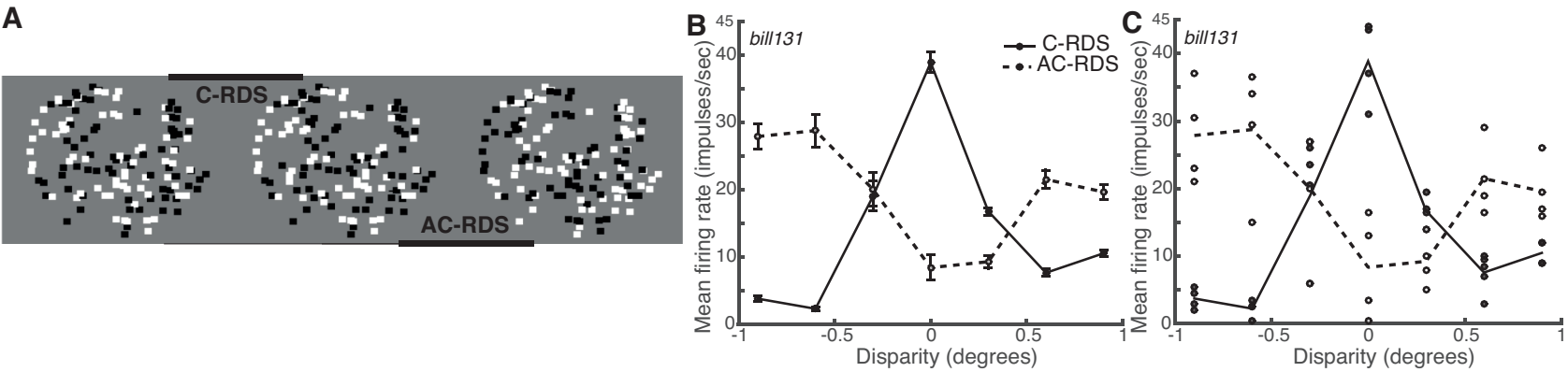

Figure 1: (A) Example correlated (C-RDS) and anti-correlated random-dot stereograms (A-RDS). A C-RDS is formed when the left image is presented to the left eye and the central image to the right eye: a central circular patch stands out in far depth (which can be seen under cross-eyed fusion). An AC-RDS is formed when the central image is presented to the left eye and the right image to the right eye; in these images corresponding dot contrasts are reversed. Perceptually, the AC-RDS appears rivalrous with no consistent depth. (B) Tuning curves and (C) firing rates per trial of an example neuron at site bill131 in visual area V5/MT. The tuning curve for AC-RDS is offset by a phase difference close to $\pi$. For this site, the variance of individual firing rates over trials was much greater for AC-RDS compared to C-RDS, as can be seen by comparing the vertical spread of the data points at each disparity value. Error bars show s.e.m.

of neuronal activity can be achieved by both feed-forward mechanisms, such as shunting inhibition [7], and recurrent (feed-back) networks with attractor dynamics [12].

In the present study, we examine the contribution of the variance of neuronal signals to the mechanisms of stereopsis by comparing variance/mean ratios of neuronal firing rates in three cortical areas across the visual hierarchy when presented with correlated versus anti-correlated stereograms. Our results help to extend models of cortical processing that underlie solutions to the stereo correspondence problem necessary for accurate depth judgments.

\section{METHODS}

\subsection{Subjects and electrophysiology}

Extracellular single-unit recordings were made in the visual cortex of five alert monkeys (Macaca mulatta) using tungsten microelectrodes. The following visual areas were targeted in each of two monkeys: area V1 (animals $\mathrm{Hg}$ and $\mathrm{Rb}$ ); area V2 (animals Dm and $\mathrm{Hg}$ ); area V5/MT (animals Bill and $\mathrm{Rg}$ ). Some of these neurons were re-analysed from previous studies (V1 [2]; V5/MT [6]). Visual areas were identified through well-established physiological criteria, including response properties, retinotopic organisation of receptive fields, and the depth of recording sites. Eye positions were recorded with scleral search coils implanted under general anaesthesia. All procedures were carried out in accordance with UK Home Office regulations on animal experimentation.

Spike waveforms and eye-position traces were saved to disk with a time-stamp using the Datawave Discovery package (DataWave Technologies (B)). Unit isolation was checked off-line by inspecting waveforms and re-selecting individual clusters of waveform shapes. Binocular fixation was assessed with respect to a fixed size window (usually $1-2^{\circ}$ in diameter). Each single unit response was measured as mean firing rate, summing spikes that occurred $40 \mathrm{~ms}$ post-stimulusonset to $40 \mathrm{~ms}$ post-stimulus-offset.

\subsection{Visual stimuli and task}

Random-dot stereograms (RDS; Figure 1) were presented in a Wheatstone stereoscope, using two computer monitors to display dot patterns separately to each eye. Horizontal binocular disparities were introduced by shifting the location of the dots in each eye. Stereograms consisted of equal numbers of black and white dots presented on a mid-gray background ( $25 \%$ dot density, at $99 \%$ contrast). For C-RDS, dot contrasts were the same in the left and right images, i.e., a black (white) dot in the right image corresponded to a black (white) dot in the left image. For AC-RDS, dot contrasts were reversed, i.e., a black dot in the right image corresponded to a white dot in the left image, and a white dot in the right image corresponded to a black dot in the left (Figure 1A). All RDS stimuli consisted of a background annular region at zero disparity and a foreground region whose disparity was varied from trial to trial. The sequence of disparity values was pseudo-randomized over trials. Animals were trained to maintain binocular fixation on a central dot for a fluid reward. Although animals maintained consistent fixation throughout each trial, different stimuli could be associated with different patterns of micro-saccades or vergence eye movements within the fixation window, which might add a component of variability to neuronal firing. This point requires further investigation. Stimuli were matched to the size and location of the receptive field of the recorded units and presented for $2000 \mathrm{~ms}$.

In V1 and V2 experiments, dot widths were usually $0.08^{\circ}$ and $0.2^{\circ}$, respectively, and the RDS were dynamic but not drifting in consistent motion, i.e., dots were plotted at a random new location on each refresh of the video frame. In V5/MT experiments, dot width was usually $0.25^{\circ}$ and dots moved coherently in the preferred direction of the recorded neurons. Stimulus parameters were selected to optimize neuronal responses in the respective visual areas: the use of coherent motion was relevant for V5/MT as these neurons are directionally selective and have a role in coding sensory judgments about motion [11]. Importantly, however, throughout this study we compare differences in response properties to 

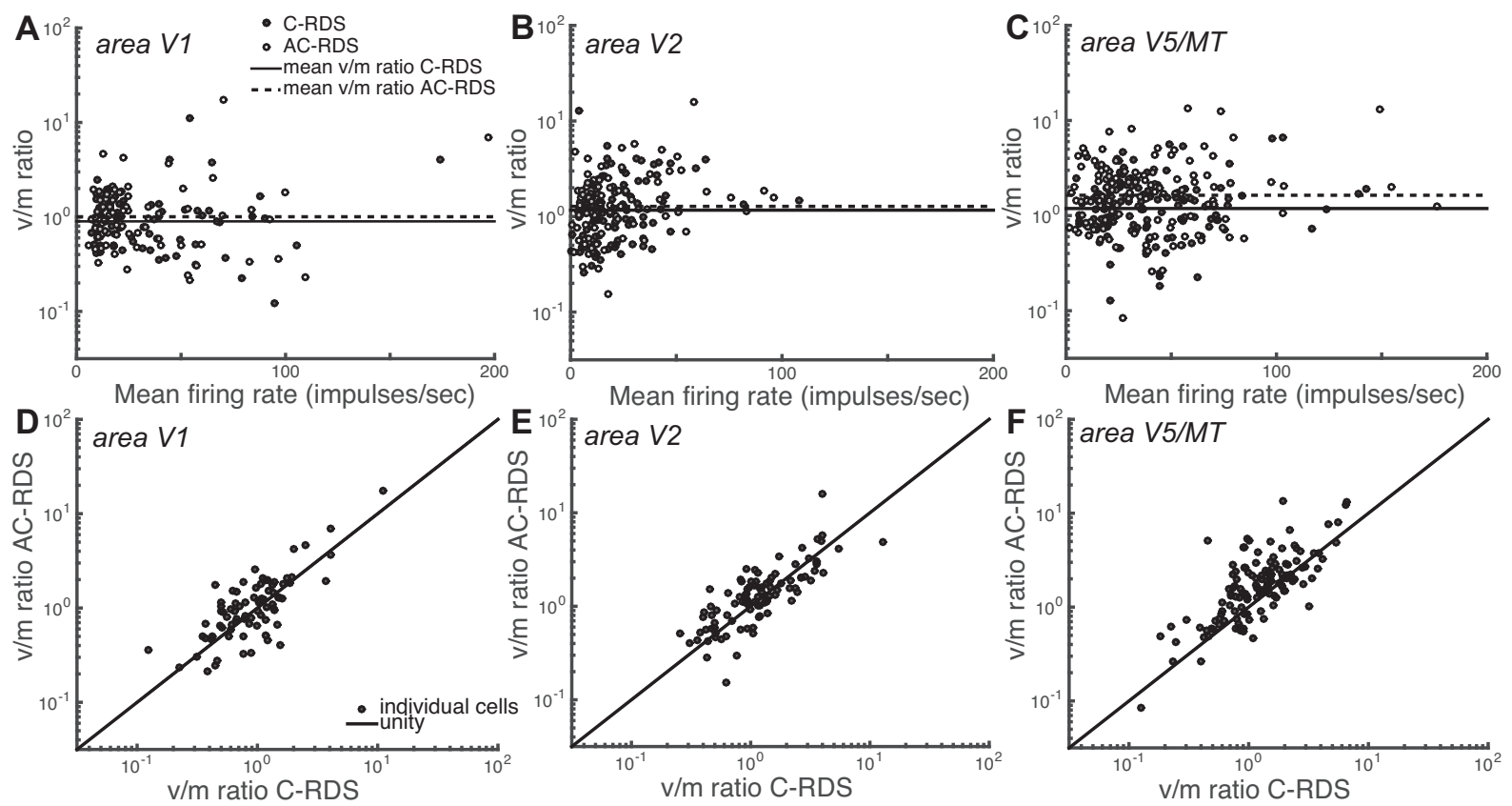

Figure 2: Variance/mean (v/m) ratios of neuronal responses to C-RDS and AC-RDS, measured in visual cortical areas V1, V2 and V5/MT. Upper panels $(\mathrm{A}, \mathrm{B}, \mathrm{C})$ show $\mathrm{v} / \mathrm{m}$ ratio versus mean firing rate, and lower panels $(\mathrm{D}, \mathrm{E}, \mathrm{F})$ show $\mathrm{v} / \mathrm{m}$ ratio for $\mathrm{AC}$-RDS plotted against $\mathrm{v} / \mathrm{m}$ ratio for $\mathrm{C}-\mathrm{RDS}$; each point represents one neuron in the visual area. V5/MT neurons tend to show larger $\mathrm{v} / \mathrm{m}$ ratios for AC-RDS than C-RDS (in general, more cells are above the line of unity) compared with from V1 and V2.

correlated versus anti-correlated versions of identical RDS patterns for every neuron tested.

\subsection{Data analysis}

Neurons were excluded from further analysis if they met any of the following criteria: no significant disparity tuning for C-RDS (ANOVA, $\mathrm{p}>0.05$ ); maximum firing rate less than 10 impulses/sec; less than 3 trials for each disparitycorrelation combination. For each included neuron, separately for correlated and anti-correlated stimuli, the mean firing rate (impulses/sec) and the firing rate variance were calculated over trials for each binocular disparity. We then calculated the variance/mean ratio $(\mathrm{v} / \mathrm{m}$ ratio) of firing response for each binocular disparity. Given that this is a ratio, the geometric mean was always used to average data and statistical tests were performed after taking logarithms. The $\mathrm{v} / \mathrm{m}$ ratios were averaged over disparities, yielding an overall $\mathrm{v} / \mathrm{m}$ ratio for correlated and another for anti-correlated stimuli for each single neuron. Paired t-tests were used to compare logarithms of the $\mathrm{v} / \mathrm{m}$ ratios for correlated and anticorrelated stimuli for each cortical area within each subject, at an alpha level of 0.05 . Average $\mathrm{v} / \mathrm{m}$ ratios for correlated and anti-correlated stimuli at each cortical area were calculated over the single neurons.

\section{RESULTS}

We calculated the variance/mean $(\mathrm{v} / \mathrm{m})$ ratio of firing rate responses to correlated and anti-correlated stereograms for neurons recorded in different visual cortical areas. For the neuron recorded at site bill131 in V5/MT, the spread of firing rates across individual trials at each disparity is generally greater for AC-RDS than C-RDS, illustrating that variance in firing rates was greater for AC-RDS (Figure 1B\&C).

Indeed, mean $\mathrm{v} / \mathrm{m}$ ratio across neurons was generally greater for AC-RDS compared to C-RDS for all subjects and visual areas, but the size of the difference, and whether it reached significance, depended upon location in the visual cortical hierarchy (Figure 2). Average $\mathrm{v} / \mathrm{m}$ ratio was 0.90 for $\mathrm{C}$ RDS and 1.01 for AC-RDS in area V1, 1.17 and 1.28 respectively in area V2, and 1.19 and 1.64 in area V5/MT. For the early visual areas V1 and V2, the difference between logtransformed $\mathrm{v} / \mathrm{m}$ ratios did not reach significance for any subject (paired t-tests; V1: $\mathrm{Hg}: \mathrm{n}=33, \mathrm{p}=0.168$; $\mathrm{Rb}: \mathrm{n}$ $=46, \mathrm{p}=0.188$; V2: Dm: $\mathrm{n}=46, \mathrm{p}=0.653 ; \mathrm{V} 2: \mathrm{Hg}: \mathrm{n}=$ $76, \mathrm{p}=0.052)$, and it did not reach significance for either V1 or V2 overall (paired t-tests; V1: $\mathrm{n}=79, \mathrm{p}=0.054$; V2: $\mathrm{n}=122, \mathrm{p}=0.062)$. However, for visual area V5/MT the difference in $\mathrm{v} / \mathrm{m}$ ratios between $\mathrm{AC}-\mathrm{RDS}$ and $\mathrm{C}-\mathrm{RDS}$ was significant for each subject individually (paired t-tests; Bill: $n=41, p<10^{-5}$; Rg: $n=99, p<10^{-4}$ ) and consequently also for area V5/MT overall $\left(n=140, p<10^{-8}\right)$.

Restricting the analysis to those neurons that showed significant tuning to AC-RDS as well as to C-RDS did not change the results of any of the statistical tests: differences in $\mathrm{v} / \mathrm{m}$ ratios remained non-significant for areas V1 and V2 $(n=45, p=0.372$ and $n=37, p=0.851)$ and significant for area V5/MT $\left(n=62, \mathrm{p}<10^{-4}\right)$.

\section{DISCUSSION}

Neurons in area V5/MT, but not V1 or V2, had a significantly greater $\mathrm{v} / \mathrm{m}$ ratio for anti-correlated compared to 
correlated stereo-stimuli. These results demonstrate greater response variability across trials to anti-correlated stimuli, which do not give rise to a coherent stereo percept, in area V5/MT compared with earlier areas V1 and V2.

\subsection{Implications for models of stereopsis}

For every neuron tested in this study, the mathematical specification of all the parameters (dot density, dot number, refresh rate, etc.) of the C-RDS and AC-RDS stimuli was identical, except for the change in contrast of the dots presented to each eye. Any variability of neuronal firing that is driven in part by variability in the temporal arrival of the dots forming the stimulus is therefore identical for the two types of stereo stimuli. Importantly, within each visual area the comparison was made between correlated and anticorrelated versions of the same stimulus, thus controlling for the RDS pattern. This allows us to draw conclusions about the effect of AC-RDS on neuronal responses with stimuli that, in correlated form, effectively drive firing.

Since neurons in visual area V5/MT are best driven by coherently moving visual stimuli, and are thought to code perceptual judgments of motion [11], as well as depth, the RDS presented in V5/MT experiments drifted coherently in the preferred direction of the recorded neurons. For areas V1 and V2, where there are fewer direction-selective cells, RDS stimuli were dynamic but did not drift in constant motion.

Regardless of the arrangement of dots forming the RDS, the perceptual appearance of C-RDS comprises a coherent set of binocularly matched dots forming a single surface in depth. By comparison, many of the dots in AC-RDS remain unfused binocularly, sometimes leading to an apparent difference in perceived dot density between C-RDS and AC-RDS. With an AC-RDS, many of the unpaired dots are effectively monocular but are nonetheless visible perceptually. If these unpaired dots contribute to the variability of neuronal firing, this additional variability should only be observable at higher stages of processing where the correspondence problem is partly solved and in particular at stages where false matches in C-RDS patterns are rejected. At early stages of binocular processing, in V1 and V2, where there appears to be local processing of disparities without a solution of the correspondence problem, there is no additional variability, since AC-RDS and C-RDS are locally identical apart from the change in contrast. Our results support the view that the solution to the stereo correspondence problem takes place at higher but not lower levels of the visual cortical hierarchy [4], and further suggest that where false matches represented at higher levels of processing, they are associated with increased neural variability.

Recent investigations spanning many cortical areas across different species have shown that the onset of a stimulus quenches neural variability in a manner that suggests that cortical circuits become more stable when driven [1]. Although, in the present study, we did not measure changes in variability at stimulus onset, our results suggest that any stimulus-induced change in variability would be equal for CRDS and AC-RDS in early visual areas, but that AC-RDS is less effective than C-RDS at quenching neural variability in area V5/MT. Why should AC-RDS be less effective for area V5/MT? Proposed mechanisms for network stabilisation in- clude bottom-up sources, such as shunting inhibition [7], and top-down sources, such as large recurrent (feed-back) networks with attractor dynamics [12]. Since AC-RDS contributes to the quenching of variability in early but not later visual areas, this suggests that the stabilizing influence does not feed forward from early visual areas to area V5/MT. Instead, V5/MT may be part of a recurrent cortical network that processes stereo images and is stabilized only if and when a global solution to the correspondence problem is reached. If a global solution cannot be found, as in the case of AC-RDS, a larger variability in firing may reflect a failure to reach a fixed point within the attractor dynamics. The activity of such a recurrent network could feasibly implement a top-down signal that allows V5/MT neurons to contribute to depth perception in a task-specific way [6].

\section{ACKNOWLEDGMENTS}

This work was supported by the Wellcome Trust. KK is a Royal Society University Research Fellow. We thank O.M. Thomas and A.K. Allouni, who collected the V2 datasets.

\section{REFERENCES}

[1] M. M. Churchland et al. Stimulus onset quenches neural variability: a widespread cortical phenomenon. Nat. Neurosci., 13(3):369-78, 2010.

[2] B. G. Cumming and A. J. Parker. Responses of primary visual cortical neurons to binocular disparity without depth perception. Nature, 389:280-283, 1997.

[3] J. V. Dodd, K. Krug, B. G. Cumming, and A. J. Parker. Perceptually bistable three-dimensional figures evoke high choice probabilities in cortical area MT. $J$. Neurosci., 21(1):4809-21, 2001.

[4] P. Janssen, R. Vogels, and G. A. Orban. At least at the level of inferior temporal cortex, the stereo correspondence problem is solved. Neuron, 37(4):693-701, 2003.

[5] K. Krug, N. Cicmil, A. J. Parker, and B. G. Cumming. A causal role for V5/MT neurons coding motion-disparity conjunctions in resolving perceptual ambiguity. Curr. Bio;., 23:1454-59, 2013.

[6] K. Krug, B. G. Cumming, and A. J. Parker. Comparing perceptual signals of single V5/MT neurons in two binocular depth tasks. $J$. Neurophysiol., 92:1586-96, 2004.

[7] C. Monier, F. Chavane, P. Baudot, and L. J. Graham. Orientation and direction selectivity of synaptic inputs in visual cortical neurons: a diversity of combinations produces spike tuning. Neuron, 37:663-80, 2003.

[8] I. Ozhawa, G. C. DeAngelis, and R. D. Freeman. Stereoscopic depth discrimination in the visual cortex: neurons ideally suited as disparity detectors. Science, 249:1037-41, 1990

[9] A. J. Parker. Binocular depth perception and the cerebral cortex. Nat. Rev. Neurosci., 8:379-91, 2007.

[10] G. E. Poggio. Mechanisms of stereopsis in monkey visual cortex. Cereb. Cortex, 5(3):193-204, May 1995.

[11] C. D. Salzman, K. H. Britten, and W. T. Newsome. Cortical microstimulation influences perceptual judgements of motion direction. Nature, 346:174-77, 1990.

[12] X. J. Wang. Decision making in recurrent neuronal circuits. Neuron, 60:215-34, 2008. 